\title{
CONNECTION RELATIONS AND QUANTIFIER SCOPE
}

\author{
Longin Latecki \\ University of Hamburg \\ Department of Computer Science \\ Bodenstedtstr. 16, 2000 Hamburg 50, Germany \\ e-mail: latecki@rz.informatik.uni-hamburg.dbp.de
}

\begin{abstract}
A formalism will be presented in this paper which makes it possible to realise the idea of assigning only one scope-ambiguous representation to a sentence that is ambiguous with regard to quantifier scope. The scope determination results in extending this representation with additional context and world knowledge conditions. If there is no scope determining information, the formalism can work further with this scope-ambiguous representation. Thus scope information does not have to be completely determined.
\end{abstract}

\section{INTRODUCTION}

Many natural language sentences have more than one possible reading with regard to quantifier scope. The most widely used methods for scope determination generate all possible readings of a sentence with regard to quantifier scope by applying all quantifiers which occur in the sentence in all combinatorically possible sequences. These methods do not make use of the inner structure and meaning of a quantifier. At best, quantifiers are constrained by external conditions in order to eliminate some scope relations. The best known methods are: determination of scope in LF in GB (May 1985),
Cooper Storage (Cooper 1983, Keller 1988) and the algorithm of Hobbs and Shieber (Hobbs/Shieber 1987). These methods assign, for instance, six possible readings to a sentence with three quantifiers. Using these methods, a sentence must be disambiguated in order to receive a semantic representation. This means that a scope-ambiguous sentence necessarily has several semantic representations, since the formalisms for the representation do not allow for scope-ambiguity.

It is hard to imagine that human beings disambiguate scope-ambiguous sentences in the same way. The generation of all possible combinations of sequences of quantifiers and the assignment of these sequences to various readings seems to be cognitively inadequate. The problem becomes even more complicated when natural language quantifiers can be interpreted distributively as well as collectively, which can also lead to further readings. Let us take the following sentence from Kempson/Cormack (1981) as an example:

Two examiners marked six scripts.

The two quantifying noun phrases can in this case be interpreted either distributively or collectively. The quantifier two examiners can have wide scope over the quantifier six scripts, or vice versa, which all in all can lead to various readings. Kempson and Cormack assign four possible readings to this sentence, 
Davies (1989) even eight. (A detailed discussion will follow.) No one, however, will make the claim that people will first assign all possible representations with regard to the scope of the quantifiers and their distribution, and will then eliminate certain interpretations according to the context; but this is today's standard procedure in linguistics. In many cases, it is also almost impossible to determine a preferred reading. The difficulties that people have when they are forced to disambiguate such sentences (to explicate all possible readings) point to the fact that people only assign an underdetermined scope-ambiguous representation in the first place.

Such a representation of the example sentence would only contain the information that we are dealing with a marking-relation between examiners and scripts, and that we are always dealing with two examiners and six scripts. This representation does not contain any information about scope. On the basis of this representation one may in a given context derive a representation with a determined scope. But it may also be the case that this information is sufficient in order to understand the sentence if no scope-defining information is given in the context, since in many cases human beings do not disambiguate such sentences at all. They use underdetermined, scopeless interpretations, because their knowledge often need not be so precise. If a disambiguation is carried out, then this process is done in a very natural way on the basis of context and world knowledge. This points to the assumption that scope determination by human beings is performed on a semantic level and is deduced on the basis of acquired knowledge.

I will present a formalism which works in a similar way. This formalism will also show that it is not necessary to work with many sequences of quantifiers in order to determine the various readings of a sentence with regard to quantifier scope.

Within this formalism it is possible to represent an ambiguous sentence with an ambiguous representation which need not be disambiguated, but can be disambiguated at a later stage. The readings can either be specified more clearly by giving additional conditions, or they can be deduced from the basic ambiguous reading by inference. Here, the inner structure and the meaning of quantifiers play an important role. The process of disambiguation can only be performed when additional information that restricts the number of possible readings is available. As an example of such information, I will treat anaphoric relations.

Intuitively speaking, the difference between assigning an undertermined representation to an ambiguous sentence and assigning a disjunction of all possible readings to this sentence corresponds to the difference between the following statements":

"Peter owns between 150 and 200 books."

and

"Peter owns 150 or 151 or 152 or ... or 200 books."

It goes without saying that both statements are equivalent, since we can understand " 150 or 151 or ... or 200 " as a precise specification of "between 150 and 200". Nevertheless, there are procedural differences in processing the two pieces of information; and there are cognitive differences for human beings, since we would never explicitly utter the second sentence. If we could represent "between 150 and 200" directly by a simple formula and not by giving a disjunction of 51 elements, then we may certainly gain great procedural and representational advantages.

The deduction of readings in semantics does not of course exclude a consideration of syntactic restrictions. They can be imported into the semantics, for example by passing syntactic information with special indices, as

* The comparison stems from Christopher Habel. 
described in Latecki (1991). Nevertheless, in this paper I will abstain from taking syntactic restrictions into consideration.

\section{SCOPE-AMBIGUOUS REPRESENTATION AND SCOPE DETERMINATION}

The aims of the representation presented in this paper are as follows:

1. Assigning an ambiguous semantic representation to an ambiguous sentence (with regard to quantifier scope and distributivity), from which further readings can later be inferred.

2. The connections between the subject and objects of a sentence are explicitly represented by relations. The quantifiers (noun phrases) constitute restrictions on the domains of these relations.

3. Natural language sentences have more than one reading with regard to quantifier scope (and distributivity), but these readings are not independent of one another. The target representation makes the logical dependencies of the readings easily discernible.

4. The construction of complex discourse referents for anaphoric processes requires the construction of complex sums of existing discourse referents. In conventional approaches, this can lead to a combinatorical explosion (cf. Eschenbach et al. 1989 and 1990). In the representation which is presented here, the discourse referents are immediately available as domains of the relations. Therefore, we need not construe any complex discourse referents. Sometimes we have to specify a discourse referent in more detail, which in turn can lead to a reduction in the number of possible readings.

I now present the formalism.

The representational language used here is second-order predicate logic. However, I will mainly use set-theoretical notation (which can be seen as an abbreviation of the corresponding notation of second-order logic). I choose this notation because it points to the semantic content of the formulas and is thus more intuitive.

Let $R \subseteq X \times Y$ be a relation, that means, a sub-set of the product of the two sets $X$ and $Y$. The domains of $R$ will be called Dom $R$ and Range $R$, with

Dom $R=\{x \in X: \exists y \in Y R(x, y)\}$ and Range $R=\{y \in Y: \exists x \in X R(x, y)\}$.

I make the explicit assumption here that all relations are not empty. (This assumption only serves in this paper to make the examples simpler.)

In the formalism, a verb is represented by a relation whose domain is defined by the arguments of verbs. Determiners constitute restrictions on the domains of the relation. These restrictions correspond to the role of determiners in Barwise's and Cooper's theory of generalized quantifiers (Barwise and Cooper 1981). This means for the following sentence:

\section{(1.1) Every boy saw a movie.}

that there is a relation of seeing between boys and movies.

In the formal notation of second-order logic we can describe this piece of information as follows:

$$
\begin{gathered}
\text { (1.1.a) } \exists X 2(\forall x y(X 2(x, y) \rightarrow \operatorname{Saw}(x, y) \& \\
\operatorname{Boy}(x) \& \operatorname{Movie}(y)))
\end{gathered}
$$

$\mathrm{X} 2$ is a second-order variable over the domain of the binary predicates; and Saw, Boy, and Movie are second-order constants which represent a general relation of seeing, the set of all boys, and the set of all movies, respectively. We will abbreviate the above formula by the following set-theoretical formula: 


\section{(1.1.b) $\exists$ saw (saw $\subseteq$ Boy $\times$ Movie)}

In this formula, we view saw as a sorted variable of the sort of the binary seeingrelations. The variable saw corresponds to the variable $\mathrm{X} 2$ in (1.1.a).

(1.1.b) describes an incomplete semantic representation of sentence (1.1). Part of the certain knowledge that does not determine scope in the case of sentence (1.1) is also the information that all boys are involved in the relation, which is easily describable as:

Dom saw=Boy. We obtain this information from the denotation of the determiner every. In this way we have arrived at the scopeambiguous representation of (1.1):

\section{(1.1.c) $\exists$ saw (saw $\subseteq$ Boy $\times$ Movie \& Dom saw $=$ Boy)}

It may be that the information presented in (1.1.c) is sufficient for the interpretation of sentence (1.1). A precise determination of quantifier scope need not be important at all, since it may be irrelevant whether each boy saw a different movie (which corresponds to the wide scope of the universal quantifier) or whether all boys saw the same movie (which corresponds to the wide scope of the existential quantifier).

Classic procedures will in this case immediately generate two readings with definite scope relations, whose notations in predicate logic are given below.

(1.2.a) $\forall x(\operatorname{boy}(x) \rightarrow \exists y(\operatorname{movie}(y) \& \operatorname{saw}(x, y)))$

(1.2.b) $\exists y(\operatorname{movie}(y) \& \forall x(\operatorname{boy}(x) \rightarrow \operatorname{saw}(x, y)))$

We can also obtain these representations in our formalism by simply adding new conditions to (1.1.c), which force the disambigiuation of (1.1.c) with regard to quantifier scope. To obtain reading (1.2.b), we must come to know that there is only one movie, which can be formaly writen by $\mid$ Range saw $\mid=1$, where $\mid$. | denotes the cardinality function. To obtain reading (1.2.a) from (1.1.c), we do not need any new information, since the two formulas are equivalent. This situation is due to the fact that (1.2.b) implies (1.2.a), which means that (1.2.b) is a special case of (1.2.a). This relation can be easly seen by comparing the resulting formulas, which correspond to readings (1.2.a) and (1.2.b):

\section{(1.3.a) $\exists$ saw (saw $\subseteq$ Boy $\times$ Movie \&} Dom saw=Boy)

\section{(1.3.b) $\exists$ saw (saw $\subseteq$ Boy $\times$ Movie \& Dom saw=Boy \& $\mid$ Range saw $\mid=1$ )}

So, we have $(1 \cdot 3 \cdot b)=>(1.3 . a)$.

As I have stated above, however, it is not very useful to disambiguate representation (1.1.c) immediately. It makes more sense to leave representation (1.1.c) unchanged for further processing, since it may be that in the development a new condition may appear which determines the scope. For instance, we can obtain the additional condition in (1.3.b), when sentence (1.1) is followed by a sentence containing a pronoun refering to a movie, as in sentence (1.4).

(1.4) It was "Gone with the Wind".

Since it refers to a movie, the image of the saw-relation (a subset of the set of movies) can contain only one element. Thus, the resolution of the reference results in an extension of representation (1.1.c) by the condition

$\mid$ Range saw $\mid=1$. Therefore, we get in this case only one reading (1.3.b) as a representation of sentence (1.1), which corresponds to wide scope of the existential quantifier. Thus in the context of (1.4) we have disambiguated sentence (1.1) with regard to quantifier scope without having first generated all possible readings (in our case these were (1.2.a) and (1.2.b)). 
Let us now assume that sentence (1.5) follows (1.1).

\section{(1.5) All of them were made by Walt Disney Studios.}

Syntactic theories alone are of no help here for finding the correct discourse referent for them in sentence (1.1), since there is no number agreement between them and a movie. The plural noun them, however, refers to all movies the boys have seen. This causes great problems for standard anaphora theories and plural theories, since there is no explicit object of reference to which them could refer (cf. Eschenbach et al. 1990; Link 1986). Thus, the usual procedure would be to construe a complex reference object as the sum of all movies the boys have seen. With my representation, we do not need such procedures because the discourse referents are always available, namely as domains of the relations. In the context of (1.1) and (1.5), the pronoun them (just as it in (1.4)) refers to the image of the relation saw, which additionally serves the purpose of determining the quantifier scope. Here, just as in the preceding cases, the representation (1.1.c) has to be seen as the "starting representation" of (1.1). The information that them is a plural noun is represented by the condition |Range saw $\mid>1$, which in turn leads to the following representation:

\section{(1.6) $\exists$ saw (saw $\subseteq$ Boy $\times$ Movie \& Dom saw $=$ Boy \& $\mid$ Range saw $\mid>1$ )}

The representation (1.6) is not ambiguous with regard to quantifier scope. The universal quantifier has wide scope over the whole sentence, due to the condition |Range saw $\mid>1$. The reading presented in (1.6) is a further specification of (1.3.a), which at the same time excludes reading (1.3.b). Thus (1.6) contains more information that formula (1.2.a), which is equivalent to (1.3.a).
A classical scope determining system can only choose one of the readings (1.2.a) and (1.2.b). However, if it chooses (1.2.a), it will not win any new information, since (1.2.b) is a special case of (1.2.a). So, quantifier scope can not be completely determined by such a system.

In order to indicate further advantages of this representation formalism, let us take a look at the following sentence (cf. Link 1986):

\section{(1.7) Every boy saw a different movie.}

Its representation is generated in the same way as that of (1.1), the only difference being that the word different carries additional information about the relation saw. different requires that the relation be injective. Therefore, the formula (1.1.c) is extended by the condition 'saw is $1-1$ '. The formula (1.8) thus represents the only reading of sentence (1.7), in which scope is completely determined; the universal quantifier has wide scope.

\section{(1.8) $\exists$ saw (saw $\subseteq$ Boy $\times$ Movie \& Dom saw=Boy \& saw is 1-1)}

\section{SCOPE-AMBIGUOUS REPRESENTATION FOR SENTENCES WITH NUMERIC QUANTIFIERS}

So far, I have not stated exactly how the representation of sentence (1.1) was generated. In order to do so, let us take an example sentence with numeric quantifiers:

\section{(2.1) Two examiners marked six scripts.}

It is certainly not a new observation that this sentence has many interpretations with regard to quantifier scope and distributivity, which can be summarized to a few main readings. However, their exact number is controversial. While Kempson and Cormack 
(1981) assign four readings to this sentence (see also Lakoff 1972), Davies (1989) assigns eight readings to it. I quote here the readings from (Kempson/Cormack 1981):

$1^{0}$ There were two examiners, and each of them marked six scripts (subject noun phrase with wide scope). This interpretation could be true in a situation with two examiners and $\mathbf{1 2}$ scripts.

$2^{0}$ There were six scripts, and each of these was marked by two examiners (object noun phrase with wide scope). This interpretation could be true in a situation with twelve examiners and six scripts.

$3^{0}$ The incomplete group interpretation: Two examiners as a group marked a group of six scripts between them.

$4^{0}$ The complete group interpretation: Two examiners each marked the same set of six scripts.

Kempson and Cormack represent these readings with the help of quantifiers over sets in the following way:

$$
\begin{aligned}
& 1^{0}\left(\exists X_{2}\right)\left(\forall x \in X_{2}\right)\left(\exists S_{6}\right)\left(\forall s \in S_{6}\right) M x s \\
& 2^{0}\left(\exists S_{6}\right)\left(\forall s \in S_{6}\right)\left(\exists X_{2}\right)\left(\forall x \in X_{2}\right) M x s \\
& 3^{0}\left(\exists X_{2}\right)\left(\exists S_{6}\right)\left(\forall x \in X_{2}\right)\left(\forall s \in S_{6}\right) M x s \\
& 4^{0}\left(\exists X_{2}\right)\left(\exists S_{6}\right)\left(\forall x \in X_{2}\right)\left(\exists s \in S_{6}\right) M x s \& \\
& \quad\left(\forall s \in S_{6}\right)\left(\exists x \in X_{2}\right) M x s
\end{aligned}
$$

Here, $X_{2}$ is a sorted variable which denotes a two-element set of examiners, and $\mathrm{S}_{6}$ is a sorted variable that denotes a six-element set of scripts.

Kempson and Cormack derive these readings from an initial formula in the conventional way by changing the order and distributivity of quantifiers. This fact is discernible from their derivational rules and the following quotation:

Generalising:

Replace ' $\left(\exists x \in X_{n}\right)^{\prime}$ by ' $\left(\forall x \in X_{n}\right)^{\prime}$
Uniformising:

Replace ' $\left(\forall x \in X_{n}\right)(\exists Y)^{\prime}$ by ' $(\exists Y)\left(\forall x \in X_{n}\right)^{\prime}$

"What we are proposing, then, as an alternative to the conventional ambiguity account is that all sentences of a form corresponding to (42) [here: 2.1] have a single logical form, which is then subject to the procedure of generalising and uniformising to yield the various interpretations of the sentence in use." (Kempson/Cormack (1981), p. 273)

Only in reading $4^{0}$ the relation between examiners and scripts is completely characterized. For the other formulas there are several possible assignments between examiners and scripts which make these formulas valid.

At this point I want to make an important observation, namely that these four readings are not totally independent of one another. I am, however, not concerned with logical implications between these readings alone, but rather with the fact that there is a piece of information which is contained in all of these readings and which does not necessitate a determinated quantifier scope. This is the information which - cognitively speaking - can be extracted from the sentence by a listener without determining the quantifier scope. The difficulties which people have when they are forced to disambiguate a sentence containing numeric quantifiers such as (2.1) without a specific context point to the fact that only such a scopeless representation is assigned to the sentence in the first place. On the basis of this representation one can then, within a given context, derive a representation with a definite scope. We can describe the scopeless piece of information of sentence (2.1), which all readings have in common, as follows. We know that we are dealing with a marking- 
relation between examiners and scripts, and that we are always dealing with two examiners or with six scripts. In the formalism described in this paper this piece of information is represented as:

\section{(2.2) $\exists$ mark ( mark $\subseteq$ Examiner $\times$ Script \& ( $\mid$ Dom mark $|=2 \vee|$ Range mark $\mid=6)$ )}

It may be that this piece of information is sufficient in order to understand sentence (2.1). If there is no scope-determining information in the given context, people can understand the sentence just as well. If, for example, we hear the following utterance,

\section{(2.3) In preparation for our workshop, two examiners corrected six scripts.}

it may be without any relevance what the relation between examiners and scripts is exactly like. The only important thing may be that the examiners corrected the scripts and that we have an idea about the number of examiners and the number of scripts.

Therefore, we have assigned an underdetermined scope-ambiguous representation (2.2) to sentence (2.1), which constitutes the maximum scopeless content of information of this sentence. The lower line of (2.2) represents a scope-neutral part of the information which is contained in the meaning of the quantifiers two examiners and six scripts. This fact indicates that the meaning of a quantifier has to be structured internally, since a quantifier contains scope-neutral as well as scopedetermining information. Distributivity is an example of scope-determining information.

Then what happens in a context which contains scope-determining information? This context just provides restrictions on the domains of the relation. These restrictions in turn contribute to scope determination. We may, for instance, get to know in a given context that there were twelve scripts in all, which excludes the condition |Range mark $\mid=6$ in the disjunction of (2.2). We then know for certain that there were two examiners and that each of them marked six different scripts. Consequently, the quantifier two examiners acquires wide scope, and we are dealing with a distributive reading. Thus, in this context we have completely disambiguated sentence (2.1) with regard to quantifier scope; and that simply on the basis of the scopeless, incomplete representation (2.2). On the other hand, standard procedures (the most important were listed at the beginning) first have to generate all representations of this sentence by considering all combinatorically possible scopes together with distributive and collective readings.

\section{CONCLUDING REMARKS}

A cognitively adequate method for dealing with sentences that are ambiguous with regard to quantifier scope has been described in this paper. An underdetermined scope-ambiguous representation is assigned to a scope-ambiguous sentence and then extended by additional conditions from context and world knowledge, which further specify the meaning of the sentence. Scope determination in this procedure can be seen as a mere byproduct. The quantifier scope is completely determined when the representation which was generated in this way corresponds to an interpretation with a fixed scope. Of course, this only works if there is scope-determining information; if not, one continues to work with the scope-ambiguous representation.

I use the language of second-order predicate logic here, but not the whole secondorder logic, since I need deduction rules for scope derivation, but not deduction rules for second-order predicate logic (which cannot be completely stated). One could even use the formalism for scope determination alone and then translate the obtained readings into a first-order formalism. However, the formalism lends itself very easily to 
representation and processing of the derived semantic knowledge as well.

\section{ACKNOWLEDGMENTS}

I would like to thank Christopher Habel, Manfred Pinkal and Geoff Simmons.

\section{BIBLIOGRAPHY}

Barwise, Jon / Cooper, Robin (1981): Generalized Quantifiers and Natural Language. Linguistics and Philosophy 4, 159-219.

Cooper, Robin (1983): Quantification and Semantic Theory. D. Reidel, Dordrecht: Holland.

Davies, Martin (1989): "Two examiners marked six scripts.' Interpretations of Numerically Quantified Sentences. Linguistics and Philosophy 12, 293-323.

Eschenbach, Carola / Habel, Christopher / Herweg, Michael / Rehkämper, Klaus (1989): Remarks on plural anaphora. Proceedings of the EACL in Manchester, England.

Eschenbach, Carola / Habel, Christopher / Herweg, Michael / Rehkämper, Klaus (1990): Rekonstruktion für plurale Diskursanaphern. In S. Felix at al. (eds.): Sprache und Wissen. Westdeutscher Verlag, Opladen.

Habel, Christopher (1986): Prinzipien der Referentialität. Imformatik Fachberichte 122. Springer-Verlag, Berlin.

Habel, Christopher (1986a): Plurals, Cardinalities, and Structures of Determination. Proceedings of COLING-86.

Hobbs, Jerry R. / Shieber, Stuart M. (1987): An Algorithm for Generating Quantifier Scopings. Computational Linguistics, Volume 13, Numbers 1-2.

Kadmon, Nirit (1987): Asymmetric Quantification. In Groenendijk, J. / Stokhof, M. / Veltman, F. (eds.):
Proceedings of the Sixth Amsterdam Colloquium.

Keller, William R. (1988): Nested Cooper Storage: The Proper Treatment of Quantification in Ordinary Noun Phrases. In U. Reyle and C. Rohrer (eds.), Natural Language Parsing and Linguistic Theories, 432-447, D. Reidel, Dordrecht.

Kempson, Ruth M. / Cormack, Annabel (1981): Ambiguity and Quantification. Linguistics and Philosophy 4, 259-309.

Lakoff, George (1972): Linguistics and Natural Logic. In Harman, G. and Davidson, D. (eds.): Semantics of Natural Language. Reidel, 545-665.

Latecki, Longin (1991): An Indexing Technique for Implementing Command Relations. Proceedings of the EACL in Berlin.

Link, Godehard (1983): The logical analysis of plurals and mass terms: A latticetheoretical approach. In Baeuerle, R. et al. (eds.), Meaning, Use, and Interpretation of Language. de Gruyter, Berlin, 302-323.

Link, Godehard (1986): Generalized Quantifiers and Plurals. In Gärdenfors, P. (ed.): Generalized Quantifiers: Studies in Lingusitics and Philosophy. Dordrrecht, The Netherlands, Reidel.

May, Robert (1985): Logical form. Its Structure and Derivation. Linguistic Inquiry Monographs. The MIT Press: Cambridge Massachusetts. 